\title{
Hydrodynamic properties of cyclodextrin molecules in dilute solutions
}

\author{
Georges M. Pavlov $\cdot$ Evguenia V. Korneeva • \\ Natalia A. Smolina • Ulrich S. Schubert
}

Received: 12 November 2008/Revised: 1 December 2008/Accepted: 8 December 2008/Published online: 22 January 2009

(C) The Author(s) 2009. This article is published with open access at Springerlink.com

\begin{abstract}
Three well-known representatives of the cyclodextrin family were completely characterized by molecular hydrodynamics methods in three different solvents. For the first time the possibility of an estimation of velocity sedimentation coefficients $s$ between 0.15 and $0.5 \mathrm{~S}$ by the numerical solution of the Lamm equation is shown. Comparison of the experimental hydrodynamic characteristics of the cyclodextrins with theoretical calculations for toroidal molecules allows an estimation of the thickness of the solvent layers on the surface of cyclodextrin molecules.
\end{abstract}

AUC\&HYDRO 2008-Contributions from 17th International Symposium on Analytical Ultracentrifugation and Hydrodynamics, Newcastle, UK, 11-12 September 2008.

G. M. Pavlov $(\bowtie)$ · U. S. Schubert

Laboratory of Macromolecular Chemistry and Nanoscience,

Eindhoven University of Technology, P.O. Box 513,

5600 MB Eindhoven, The Netherlands

e-mail: g.m.pavlov@tue.nl

URL: http://www.schubert-group.com

G. M. Pavlov

Institute of Physics, St Petersburg State University,

Ulianovskaya Str. 1, 198504 St Petersburg, Russia

E. V. Korneeva

Institute of High-Molecular Compounds of Russian Academy of Science, St Petersburg, Russia

N. A. Smolina

Faculty of Biological and Soil Sciences,

St Petersburg State University, St Petersburg, Russia

U. S. Schubert

Laboratory of Organic and Macromolecular Chemistry,

Friedrich-Schiller-University Jena, Humboldtstr. 10,

07743 Jena, Germany
Keywords Cyclodextrins $1 \cdot$ Molecular hydrodynamics 2 . Toroid 3

\section{Introduction}

Cyclodextrins (CDs) are well-known objects of supramolecular chemistry and glycoscience (Dodziuk 2006; Philp and Stoddart 1996; Szejti and Osa 1996; Wenz 1994). They are products of amylopectin enzymatic destruction by the action of extracellular enzymes, cyclodextrin glycosyltransferases (CGTase, E.C. 2.4.1.19) (Qi and Zimmermann 2005). The more abundant and available of the CDs are cyclic oligosaccharides, consisting of $6,7,8$ D-glucose residues, connected with $\alpha(1 \rightarrow 4)$ links and named $\alpha-, \beta$ and $\gamma-\mathrm{CD}$, respectively. The shape of these molecules resemble a hollow truncated cone with a central cavity, containing $\mathrm{C} 3 \mathrm{H}$ and $\mathrm{C} 5 \mathrm{H}$ carbons atoms and ester-like $\mathrm{O}-4$ and $\mathrm{O}-5$ oxygen atoms. The $\mathrm{CD}$ structure provides an external hydrophilic region and a rather hydrophobic inner cavity (Bender and Komiyama 1978). The number of sugar rings defines the size of the cavity and the flexibility of the CDs. CDs and their derivatives are well-known as molecular hosts capable of including, in their cavities, different guest molecules of appropriate size, shape, and polarity via non-covalent interactions (Connors 1997; Dodziuk 2006; Harada 1997; Saenger 1980; Szejti 2004). The apolar nature of their cavities (Harada 1997) allows CDs to act as hosts preferentially for nonpolar guests, which include small molecules and surfactants (Harada 1997; GonzálezPérez et al. 2008). The interactions of CDs with natural and synthetic polymers bearing attached hydrophobic tags have also been intensively studied (Beheshti et al. 2006; Burckbuchler et al. 2006; Charlot et al. 2006; Rinaudo et al. 2005; Sabadini and Cosgrove 2003). In addition, CD 
derivatives are being used as a core in the synthesis of star-like polymer systems (Hoogenboom et al. 2006). Cyclodextrins' ability to form inclusion complexes with appropriate sized hydrophobic guest molecules is the most frequently applied property of CDs (Hedges 1998; Szejti 2004) and has been studied by spectroscopic, kinetic, and crystallographic methods (Dodziuk 2006; Saenger et al. 1998; Szejti and Osa 1996). CDs and their derivatives are considered to be potential carriers for hydrophobic pharmaceutical compounds (Loftsson and Duchene 2007; Uekama et al. 1998). However, information about the properties of cyclodextrins molecules in diluted solution is still rather scarce (Dodziuk 2006; Nakata et al. 2003; Longsworth 1953; Szejti 1998).

In this study, we present investigations on $\alpha-, \beta$ - and $\gamma$ cyclodextrin by macromolecular hydrodynamics.

\section{Materials and methods}

The CDs studied, $\alpha$-, $\beta$ - and $\gamma$-cyclodextrin, were obtained commercially from Sigma (purity $\geq 98 \%$ ) (Table 1). Velocity sedimentation, the translational diffusion, and viscosity as well as the increment of density were measured in three different solvents: water, dimethylformamide, and dimethylsulfoxide.

Sedimentation velocity experiments were performed on a Beckman XLI analytical ultracentrifuge at a rotor speed of $55,000 \mathrm{rpm}$ and at $20^{\circ} \mathrm{C}$ in Al-double-sector cells of optical path $12 \mathrm{~mm}$ using interference optics. The evaluation program Sedfit for continuous particle size distributions (Schuck 2000) was used for data analysis. The regularization method used was the Tikhonov-Philips 2nd derivative, and the confidence level ( $F$ ratio) chosen was $0.8-0.9$. By fitting for $\left(f / f_{\mathrm{sph}}\right)$ in a nonlinear regression, an estimate of the weight-average frictional ratio of all macromolecules in solution is obtained, where $f$ is the frictional ratio of the solute macromolecule and $f_{\mathrm{sph}}$ is the frictional ratio of the rigid sphere with the same "anhydrous" volume (free of solvent) as the macromolecule. The final result is the differential distribution $(\mathrm{d} c(s) / \mathrm{d} s)$ of the sample, which is named $c(s)$. It is scaled such that the area under the $c(s)$ curve between the smallest $s$ value, $s_{1}$, and the largest one, $s_{2}$, in the distribution will give the loading concentration of macromolecules between these sedimentation coefficients (expressed in number of fringes, $J$, in the case of interference optics). $J$, which is proportional to the polymer concentration in solution, was used to calculate
Table 1 Images of modeling structures, calculated molecular mass $\left(M_{\text {calc }}\right)$, inner $\left(d_{\text {ii }}\right)$ and outer $\left(d_{0 \mathrm{i}}\right)$ diameters and the values of molecular mass obtained with MALDI-TOF MS of cyclodextrin molecules

\begin{tabular}{|c|c|c|c|c|c|}
\hline $\mathrm{CD}$ & $\begin{array}{l}\text { Images of modeling } \\
\text { structures }\end{array}$ & $\begin{array}{l}M_{\text {calc }} \\
(\mathrm{g} / \mathrm{mol})\end{array}$ & $\begin{array}{l}d_{\mathrm{ii}}^{\mathrm{a}} \\
\left(10^{8} \mathrm{~cm}\right)\end{array}$ & $\begin{array}{l}d_{0 \mathrm{i}}^{\mathrm{a}} \\
\left(10^{8} \mathrm{~cm}\right)\end{array}$ & $\begin{array}{l}M \text { (MALDI) } \\
(\mathrm{g} / \mathrm{mol})\end{array}$ \\
\hline$\alpha-\mathrm{CD}\left(\mathrm{C}_{6} \mathrm{H}_{10} \mathrm{O}_{5}\right)_{6}$ & & 972.9 & $5.0-4.7$ & 14.6 & 972.4 \\
\hline$\beta-\mathrm{CD}\left(\mathrm{C}_{6} \mathrm{H}_{10} \mathrm{O}_{5}\right)_{7}$ & & $1,135.0$ & $6.5-6.0$ & 15.4 & $1,134.5$ \\
\hline$\gamma-\mathrm{CD}\left(\mathrm{C}_{6} \mathrm{H}_{10} \mathrm{O}_{5}\right)_{8}$ & & $1,297.1$ & $8.3-7.5$ & 17.5 & $1,296.6$ \\
\hline
\end{tabular}

a As measured on space-filling or CPK models (Saenger 1980; Corey and Pauling 1953) 
the refractive index increment: $(\Delta n / \Delta c)=\mathrm{J} \lambda / \mathrm{Kcl}$ (Pavlov et al. 2003), where $\lambda$ is the wavelength $(675 \mathrm{~nm}), K$ the magnifying coefficient and 1 the optical path. With $K=1$ and $l=12 \mathrm{~mm}$ we obtain: $\Delta n / \Delta c=5.625 \times 10^{-5}(\mathrm{~J} / \mathrm{c})$ and $\mathrm{c}$ in $\mathrm{g} / \mathrm{cm}^{3}$.

Translational diffusion was studied by the classical method of forming a boundary between the solution and the solvent on Tsvetkov polarizing diffusiometer (Tsvetkov 1989). The diffusion boundary was formed in glass cell of length $h=30 \mathrm{~mm}$ along the beam path. The optical system used for recording the solution-solvent boundary in diffusion analysis was a Lebedev's polarizing interferometer (Lebedev 1930). Translational diffusion coefficients were calculated from the equation:

$\sigma^{2}=\sigma_{0}^{2}+2 D t$,

where $\sigma^{2}$ is the dispersion of the diffusion boundary calculated from the maximum ordinate and the area under the diffusion curve, $\sigma_{0}^{2}$ is the zero dispersion characterizing the quality of boundary formation, and $t$ is the diffusion time. Experiments were carried out at $25^{\circ} \mathrm{C}$, and the intrinsic diffusion coefficient, which depends only on the macromolecule properties, is calculated as: $[D]=D_{0} \eta_{0} / T$.

Viscosity measurements were conducted using an Ostwald viscometer. The respective flow times, $\tau_{0}$ and $t$, were measured at $25^{\circ} \mathrm{C}$ for the solvent and polymer solutions, with relative viscosities $\eta_{\mathrm{r}}=t / \tau_{0}$. The extrapolation to zero concentration was made by using both the Huggins and Kraemer equations (Cantor and Schimmel 1980; Tsvetkov 1989) and the average values were considered as the value of intrinsic viscosity.

The density measurements were carried out in the density meter DMA 5000 (Anton Paar, Graz, Austria) according to the procedure of Kratky et al. (1973).

The cyclodextrins were investigated also by MatrixAssisted Laser Desorption/Ionisation Time-Of-Flight Mass Spectrometry (MALDI-TOF MS). MALDI-TOF MS measurements were performed with an Ultraflex III TOF/TOF (Bruker Daltonics, Bremen, Germany) equipped with a $\mathrm{Nd}$ :YAG laser and a collision cell. All spectra were measured in the positive reflector mode. For the MS/MS mode, argon was used as collision gas at a pressure of $2 \times 10^{-6}$ mbar. The instrument was calibrated prior to each measurement with an external PMMA standard from PSS Polymer Standards Services GmbH (Mainz, Germany) in the required measurement range. MS and MS/MS data were processed using PolyTools 1.0 and an isotope pattern calculator.

\section{Results and discussion}

The velocity sedimentation and isothermal translational diffusion studies were made separately in three different solvents: water, dimethylformamide (DMF) and dimethylsulfoxide (DMSO). The solubility of the CDs studied in these solvents increases in the following order: $\mathrm{H}_{2} \mathrm{O}<\mathrm{DMF}<$ DMSO. The velocity sedimentation experiments were run overnight (12-14 h), at a solute concentration $c \cong 4 \mathrm{mg} / \mathrm{mL}$. Figure 1 represents the sedimentation interference profiles of $\alpha$-cyclodextrin in DMF and in DMSO as well as the calculated distribution of the sedimentation coefficients, $c(s)$, as obtained by the use of the Sedfit program. Figure 2 shows the comparison of the normalized differential distributions for $\gamma$-CD, obtained in the different solvents. The density increment $(\Delta \rho / \Delta c)$, which is also required for the quantitative interpretation of the sedimentation data, allows the determination of the partial specific volume $(\bar{v})$. The value remained the same for different $\mathrm{CDs}$ in the same solvent and was found to be $0.667,0.632$, and $0.649 \mathrm{~cm}^{3} / \mathrm{g}$ in $\mathrm{H}_{2} \mathrm{O}$, DMF, and DMSO, respectively. The refractive index increment $(\Delta n / \Delta c)$ also remained virtually the same for different CDs in the same solvent: 0.148, 0.104, and $0.07 \mathrm{~cm}^{3} / \mathrm{g}$ in $\mathrm{H}_{2} \mathrm{O}, \mathrm{DMF}$, and DMSO, respectively. The translational diffusion was studied at an average solute concentration $c$ of $\cong 1-2 \mathrm{mg} / \mathrm{mL}$ (Fig. 3).

The values of both the sedimentation coefficient $s$ and the diffusion coefficient $D$ obtained at the concentrations given above were assumed to be equal to the values extrapolated to zero concentration. The solute concentrations practically correspond to the limiting dilution as the values of the Debye parameter $(c[\eta])$, describing dilution of a solution, is within the limits of $0.01 \leq c[\eta] \leq 0.03$. The plots of $\eta_{\mathrm{sp}} / c$ and $\ln \eta_{\mathrm{r}} / c$ as a function of $c$ allowed us to determine the intrinsic viscosity, $[\eta]$, of the cyclodextrins (Fig. 4). Table 2 represents the values of $\mathrm{s},\left(f / f_{\mathrm{sph}}\right), D$ and $[\eta]$ obtained.

The different experimental hydrodynamics measurements can be expressed as intrinsic values, $[\eta],[s],[D],[f]$, which are independent of the solvent properties (Cantor and Schimmel 1980; Tsvetkov 1989). Each of them is related to common macromolecular characteristics such as molar mass $M$ and mean-square radius of gyration $<R^{2}>$ :

$[\eta]=\Phi^{\prime}<R^{2}>^{3 / 2} / \mathrm{M}$

$[s] \equiv s_{0} \eta_{0} /\left(1-\rho_{0} v\right)=M / N_{\mathrm{A}} P^{\prime}<R^{2}>^{1 / 2}$

$[D] \equiv D_{0} \eta_{0} / T=k /[f]=k / P^{\prime}<R^{2}>^{1 / 2}$

$[f] \equiv f_{0} / \eta_{0}=P^{\prime}<R^{2}>^{1 / 2}$

with $[\eta]$ : intrinsic viscosity; $[s],[D]$, and $[f]$ : intrinsic coefficients of velocity sedimentation, translational diffusion, and translational friction, correspondingly; $N_{\mathrm{A}}$ is the Avogadro number, and $\Phi^{\prime}$ and $P^{\prime}$ are the Flory hydrodynamic parameters. These relationships have a general meaning and are valid for any type of molecules/ 

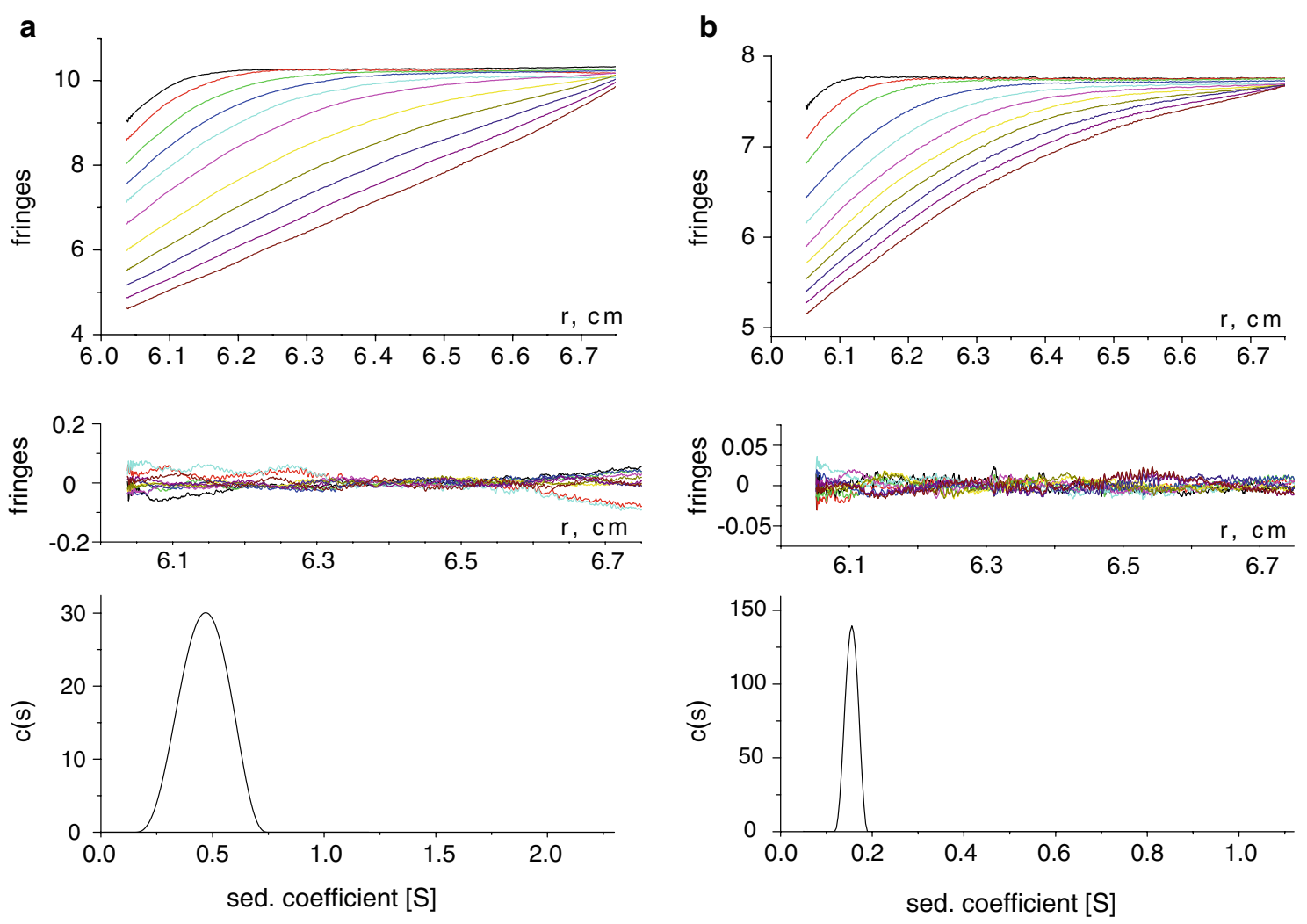

Fig. 1 Velocity sedimentation of CDs: experimental data and evaluations obtained with the Sedfit program. a $\alpha$-CD with $c=4.2 \times$ $10^{-3} \mathrm{~g} / \mathrm{cm}^{3}$ in DMF, b the same solute with $c=4.8 \times 10^{-3} \mathrm{~g} / \mathrm{cm}^{3}$ in DMSO. Panels at the top show the superposition of some interference

profiles on the whole range of sedimentation time (12 h), those at the middle the corresponding residual plots. The panels at the bottom represent the distribution of sedimentation coefficients, $c(\mathrm{~s})$, obtained with a regularization procedure with a confidence level of 0.70

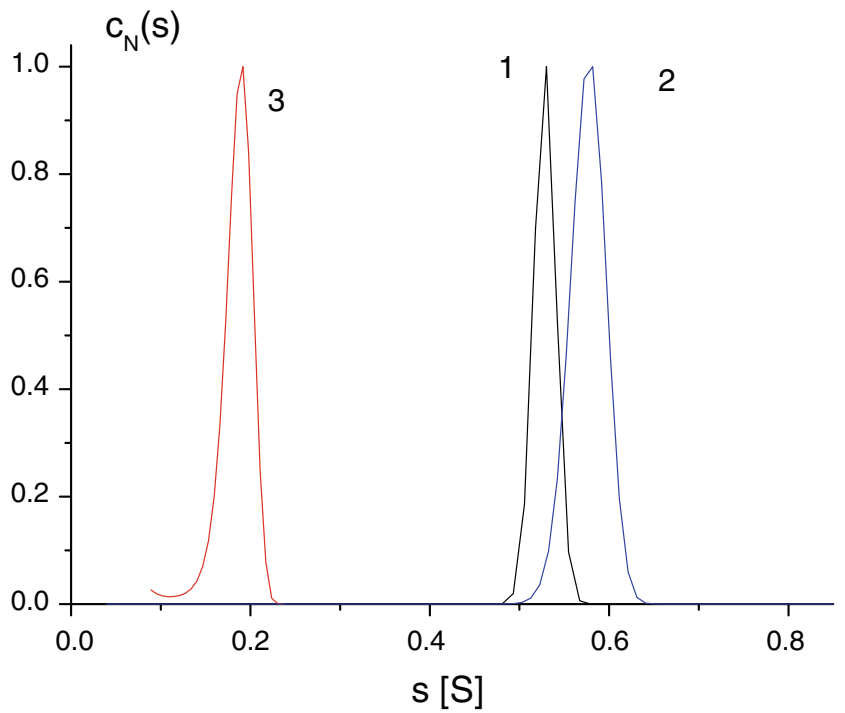

Fig. 2 Comparison of the normalized differential distributions of sedimentation coefficients, $c_{\mathrm{N}}(\mathrm{s})$, of $\gamma-\mathrm{CD}$ in $\mathrm{H}_{2} \mathrm{O}$ (1), DMF (2), and DMSO (3), as obtained with the Sedfit program. For clearness each distribution is normalized on the maximal value of $c_{\max }(\mathrm{s})$ such as $c_{\mathrm{N}}(\mathrm{s}) \equiv c(\mathrm{~s}) / c_{\max }(\mathrm{s})$ to eliminate the influence of different increments of refractive indexes macromolecules. The values of the dimensionless parameters $\Phi^{\prime}$ and $P^{\prime}$ depend, however, on the shape and asymmetry of the solute molecules and, in addition, on the hydrodynamic interactions between the different parts of the same molecule which exercise the friction in the liquid medium. The theoretical values of these parameters are obtained by solving the hydrodynamic problem and depend on the models and mathematical approximations.

A frequently used concept in biochemistry and polymer science is that of the hydrodynamic equivalent sphere. In this concept, the real molecule is modeled by a rigid sphere which has the same translational frictional coefficient. The radius of the hydrodynamic equivalent sphere is calculated from the Stokes relation $\left(5^{\prime}\right)$. In the case of the intrinsic viscosity the Einstein relation $\left(2^{\prime}\right)$ is applied.

$[f]_{\mathrm{sph}}=6 \pi R_{\mathrm{sph}}$

$[\eta]_{\mathrm{sph}}=(10 / 3) \pi N_{\mathrm{A}}\left(R_{\mathrm{sph}}^{3} / M\right)$

Obviously the radius of a hydrodynamic equivalent sphere can differ considerably from the size of the real molecule. Nevertheless, this approach is useful, in 

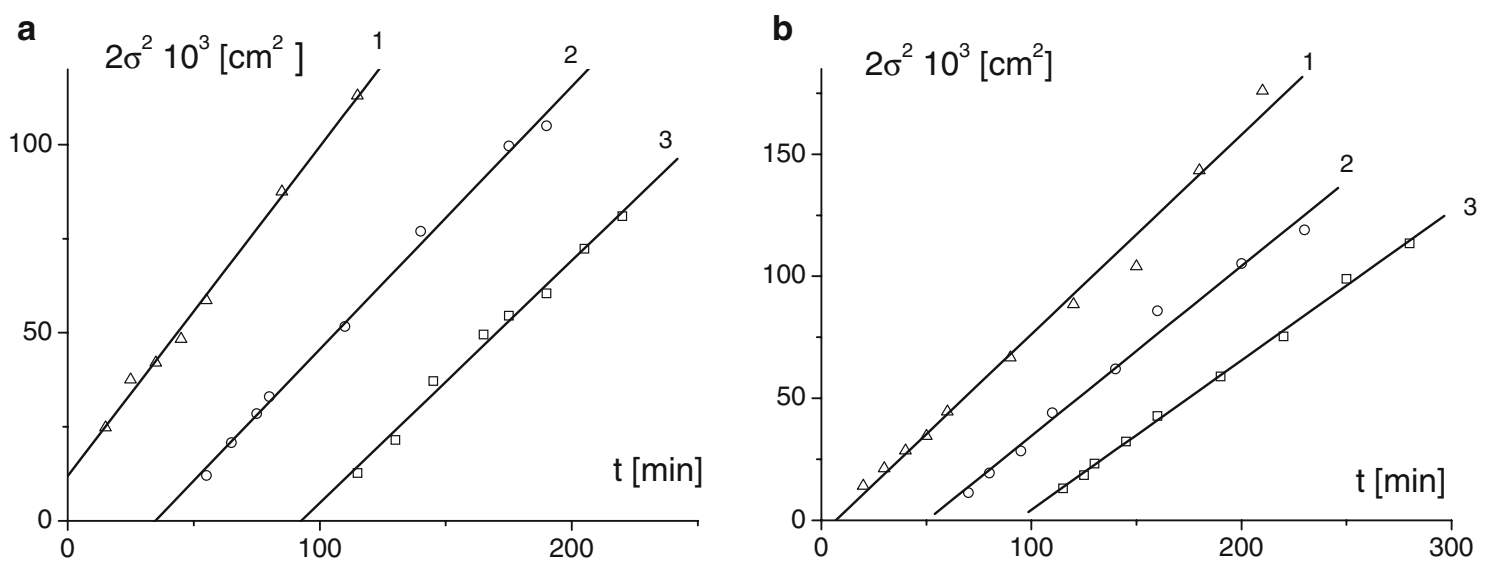

Fig. 3 Time dependence of dispersion of the diffusion boundary $2 \sigma^{2}$ versus time $t$ of diffusion in $\mathrm{H}_{2} \mathrm{O}(\mathbf{a})$ and $\mathrm{DMF}(\mathbf{b})$, for $\alpha$-CD (1), $\beta$-CD (2), and $\gamma$-CD (3)

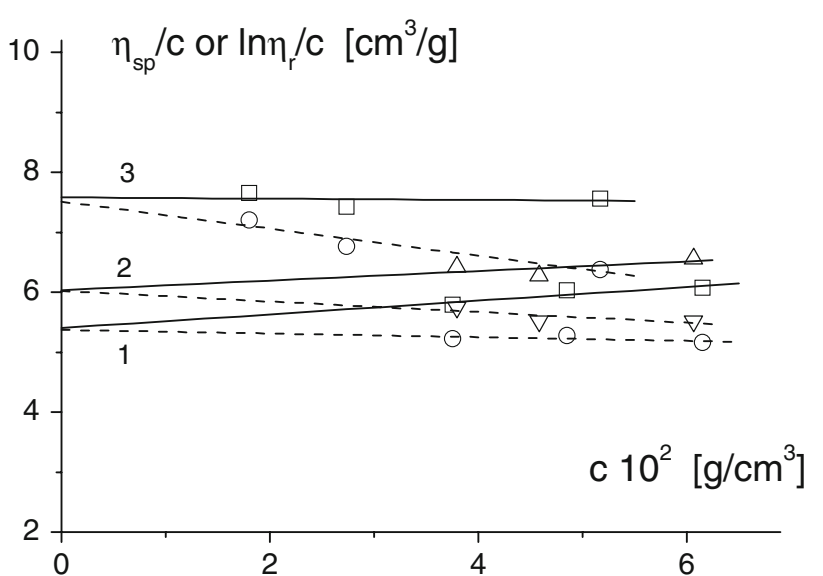

Fig. 4 Determination of the intrinsic viscosity values $[\eta]$ (which are the intercepts, at $c=0$, of the plots of $\eta_{\mathrm{sp}} / c$ (solid lines, Huggins plot) and $\ln \eta_{\mathrm{r}} / c$ [dashed lines, Kramer plot] vs. $c$ ) for $\alpha$-CD (1), $\beta$-CD (2), and $\gamma-\mathrm{CD}(3)$ in DMSO. The Huggins parameter $k_{\mathrm{H}}$ are $0.39,0.22$, and 0 , and the Kramer parameter $k_{\mathrm{K}}$ are $-0.11,-0.24$, and -0.40 for $\alpha-$, $\beta$-, and $\gamma-\mathrm{CD}$, respectively

particular when sizes in a series of polymerhomologues are to be compared, e.g., for proteins, dendrimers, or highly branched macromolecules.

Combination of Eqs. 3 and 4 leads to the Svedberg equation (Cantor and Schimmel 1980; Maechtle and Boerger 2006; Tsvetkov 1989) used for molar mass determination from the hydrodynamic data:

$M_{\mathrm{sD}}=\left(R T /\left(1-v \rho_{0}\right)\right)\left(s_{0} / D_{0}\right)=\left(N_{\mathrm{A}} /\left(1-v \rho_{0}\right)\right) s_{0} f_{0}$

The translational friction coefficient $f_{0}$ of molecules may be expressed in our case by the following way:

$f_{0}=\left(f / f_{\text {sph }}\right)_{0} f_{\text {sph }}=\left(f / f_{\text {sph }}\right)_{0} 6 \pi \eta_{0}\left(3 \mathrm{M} v_{\text {bar }} / 4 \pi N_{\mathrm{A}}\right)^{1 / 3}$

From the Eqs. 4 and 7 it is possible to calculate the translational diffusion coefficients and, correspondingly, the intrinsic translational diffusion coefficient:
$\left.[D]_{\mathrm{sf}}=k \times\left(9 \pi 2^{1 / 2}\right)^{-1}\left(\left(f / f_{\mathrm{sph}}\right)_{0}\right)^{-3 / 2}([s] v)\right)^{-1 / 2}$

Linking $s_{0}$ and $[D]_{\mathrm{sf}}$ in the equation obtained from Eqs. 6 and 8 allows us to determine the molar mass of moving molecules using:

$M_{\text {sf }}=9 \pi 2^{1 / 2} N_{\mathrm{A}}\left([s]\left(f / f_{\text {sph }}\right)_{0}\right)^{3 / 2} v^{1 / 2}$.

This equation is the transformation of original Svedberg equation 6 which, in turn, when $f / f_{\text {sph }}=1$ is transformed into the relationship describing the solid sphere model.

Table 3 shows the molar masses and hydrodynamic radii of the CDs studied, as determined from the experimental data by use of the equations given above.

Comparison with the $M$ values calculated from the chemical structure of the CDs shows that, in all solvents, the experimental data are relatively close to the theoretical ones. The measurements in DMF yield by far the closest agreement, the deviations being below $10 \%$ for all samples. But even for the worst result, obtained with $\gamma$-CD in DMSO, the deviation is below 50\%. These results are highly surprising, since the $s$ values on which they are based are around $0.5 \mathrm{~S}$ or even, in the solvent DMSO, around $0.15 \mathrm{~S}$. As far as we know, the possibility of correctly or nearly correctly determining such small velocity sedimentation coefficients on the basis of the numerical solution of the Lamm equation has not been demonstrated before. It should be noted that, in the experiments described, the evolution of the sedimentation boundary occurs without appreciable boundary displacement. Elementary estimation shows that when the sedimentation coefficient is only $0.1 \mathrm{~S}$ the shift of the sedimentation boundary during $12 \mathrm{~h}$ of experiment at $55,000 \mathrm{rpm}$ amounts to only $1 \mathrm{~mm}$. To fix this shift by usual methods against a background of significant diffusion spreading obviously is not possible. In order to further strengthen the conclusions described, 
Table 2 Translational diffusion coefficient $D$, velocity sedimentation coefficient $s$, frictional ratio $f / f_{\text {sph }}$, and intrinsic viscosity $[\eta]$, of CD in $\mathrm{H}_{2} \mathrm{O}$, DMF, and DMSO

\begin{tabular}{|c|c|c|c|c|c|c|c|c|c|c|c|}
\hline & \multicolumn{3}{|l|}{$\mathrm{H}_{2} \mathrm{O}$} & \multicolumn{4}{|l|}{ DMF } & \multicolumn{4}{|l|}{ DMSO } \\
\hline & $10^{7} D\left(\mathrm{~cm}^{2} / \mathrm{s}\right)$ & $s(\mathrm{~S})$ & $f / f_{\mathrm{sph}}$ & $10^{7} D\left(\mathrm{~cm}^{2} / \mathrm{s}\right)$ & $s(\mathrm{~S})$ & $f l f_{\mathrm{sph}}$ & {$[\eta]\left(\mathrm{cm}^{3} / \mathrm{g}\right)$} & $10^{7} D\left(\mathrm{~cm}^{2} / \mathrm{s}\right)$ & $s(\mathrm{~S})$ & $f l f_{\mathrm{sph}}$ & {$[\eta]\left(\mathrm{cm}^{3} / \mathrm{g}\right)$} \\
\hline$\alpha$ & 36.5 & 0.48 & 1.00 & 34 & 0.49 & 1.33 & 5.5 & 9.7 & 0.15 & 1.38 & 5.4 \\
\hline$\beta$ & 29.1 & 0.51 & 1.00 & 29 & 0.53 & 1.33 & 5.2 & 8.2 & 0.11 & 1.43 & 6.0 \\
\hline$\gamma$ & 27 & 0.53 & 1.06 & 25.5 & 0.58 & 1.37 & 5.9 & 8.45 & 0.18 & 1.49 & 7.5 \\
\hline
\end{tabular}

Table 3 Molar mass $(\mathrm{g} / \mathrm{mol})$ and hydrodynamic radii $R_{\mathrm{h}} \times 10^{8} \mathrm{~cm}$ $( \pm 9 \%)$ of $\mathrm{CD}$ in different solvents

\begin{tabular}{|c|c|c|c|c|c|c|c|}
\hline \multirow[t]{2}{*}{ CD } & \multicolumn{2}{|l|}{$\mathrm{H}_{2} \mathrm{O}$} & \multicolumn{2}{|l|}{ DMF } & \multicolumn{2}{|c|}{ DMSO } & \multirow[t]{2}{*}{$M_{\mathrm{av}}$} \\
\hline & $M$ & $R_{\mathrm{h}}$ & $M$ & $R_{\mathrm{h}}$ & $M$ & $R_{\mathrm{h}}$ & \\
\hline$\alpha$ & 1,100 & 6.7 & 970 & 8.2 & 1,300 & 10 & $1,100 \pm 100$ \\
\hline$\beta$ & 1,300 & 7.7 & 1,150 & 9.1 & 1,100 & 11 & $1,180 \pm 80$ \\
\hline$\gamma$ & 1,500 & 8.5 & 1,400 & 10.1 & 1,900 & 12.4 & $1,600 \pm 200$ \\
\hline
\end{tabular}

we have checked the validity of the theoretical $M$ values by MALDI-TOF. The experimental spectra are presented in Fig. 5. Taking into account the mass of sodium ion the good correlation is observed between calculated and experimental values of molar masses (Table 1).

The average values for the radii of the hydrodynamic equivalent sphere, calculated from the Stokes $\left(5^{\prime}\right)$ and Einstein $\left(2^{\prime}\right)$ relationships are also presented in Table 3. These values characterize certain external sizes of CDs molecules. When comparing these data with those measured on space-filling or CPK models (Table $1, r_{0}^{\mathrm{CPK}}=d_{0}$ l 2) (Saenger 1980; Corey and Pauling 1953), it is interesting to note that (1) both kinds of radii virtually agree in water, and (2) the ratios of the hydrodynamic to the radii $r_{0}^{\mathrm{CPK}}$ remain virtually constant in the series of studied CDs but vary in different solvents: $R_{\mathrm{H}_{2} \mathrm{O}} / r_{0}^{\mathrm{CPK}}=0.96 \pm 0.03 \cong$ $1.0, R_{\mathrm{DMF}} / r_{0}^{\mathrm{CPK}}=1.15 \pm 0.02$, and $R_{\mathrm{DMSO}} / r_{0}^{\mathrm{CPK}}=1.41 \pm$ 0.02 . Since hydrodynamic interactions inside of $\mathrm{CDs}$ molecules will contribute to the friction of the molecules, different ratios of radii in different solvents may reflect not only different sizes but also different hydrodynamic interactions.

A more adequate model for the description of the hydrodynamic behavior of CDs molecules can be toroidal particles/molecules. In recent years approaches which aimed at modeling the shape of biopolymers by bead-shell models were extended to the calculation and predictions of biopolymer hydrodynamic properties (Garcia de la Torre and Bloomfield 1977, 1981; Allison 1999; Garcia de la Torre 2001). Applying such methods to toroids, the dependency of the translational diffusion coefficient and of the intrinsic viscosity on the characteristic ratio of toroids, $x=r_{\mathrm{i}} / r_{0}$, was derived $\left(r_{\mathrm{i}}, r_{0}\right.$ : inner and outer radius, respectively) (Garcia de la Torre 2001). The following interpolating polynomials were obtained for dimensionless values $[D] r_{0} / k$ and $0.01[\eta] M /\left(N_{\mathrm{A}} r_{0}^{3}\right)$ :

$[D] r_{0} / k=0.0620-0.00143 x+0.0278 x^{2}$

$0.01[\eta] M /\left(N_{\mathrm{A}} r_{0}^{3}\right)=0.0701-0.0365 x-0.00629 x^{2}$

A comparison of the experimental and computational results is shown in Fig. 6. As a first approximation, the space-filling models or CPK models data were used to characterize the outer and inner sizes of the CD molecules (Saenger 1980; Szejti 1998). The details of the deviation of the experimental points from the theoretical curves correlate to the above-obtained estimations concerning the hydrodynamic radii of the $\mathrm{CD}$ molecules in different solvents. In the plots shown, $r_{0}$ is the more crucial parameter since the ordinate directly depends on it whereas the dependence on $r_{\mathrm{i}}$ becomes apparent only as a ratio of $r_{\mathrm{i}} / r_{0}$ in the abscissa. Both plots indicate that, in order to superimpose the experimental data to the computational results, it is necessary to increase the outer sizes of CD molecules. In practice, these sizes could be increased in solution by the absorption of a few solvent molecules to the external surface of the $\mathrm{CD}$ molecules, forming an absorbed layer. Thus, in solution the outer size of $\mathrm{CD}$ molecules may be characterized by an effective radius $r_{0}^{\text {eff }}=r_{0}+\Delta r_{0}$, where $\Delta r_{0}$ is the average thickness of solvent layer. The latter figure probably correlates to the size and the number of the solvent molecules. The size of the solvent molecules can be estimated by the relationship $d=\left(6 M / \pi \rho_{0} N_{\mathrm{A}}\right)^{1 / 3} .3 .86,6.26$, and $6.08 \times 10^{-8} \mathrm{~cm}$ for $\mathrm{H}_{2} \mathrm{O}$, DMF, and DMSO molecules were obtained, respectively. The calculated results could be fitted to the experimental ones by assuming that the thickness of the solvent layer varies depending on the solvent, amounting for $\Delta r_{0} \cong 0.5 d_{\text {solv }}$ in water and DMF but $\Delta r_{0} \cong d_{\text {solv }}$ in DMSO. These layers are formed by different numbers of solvent molecules. Qualitatively the number of DMSO molecules must be higher in comparison with both other solvents.

Although, in reality, the $\mathrm{CD}$ structure in solution are flexible, whereby CD instant conformations probably differ from each other because of a variation of the valence 
Fig. 5 MALDI-TOF-MS spectrum for the cyclodextrins $(1 \alpha$-CD, $2 \beta$-CD, $3 \gamma$-CD) obtained on dihydroxybenzoic acid as matrix and NaTFA as a source of sodium ions. The figures at the peaks are the molar masses of cyclodextrine isotopes including the mass of one sodium ion
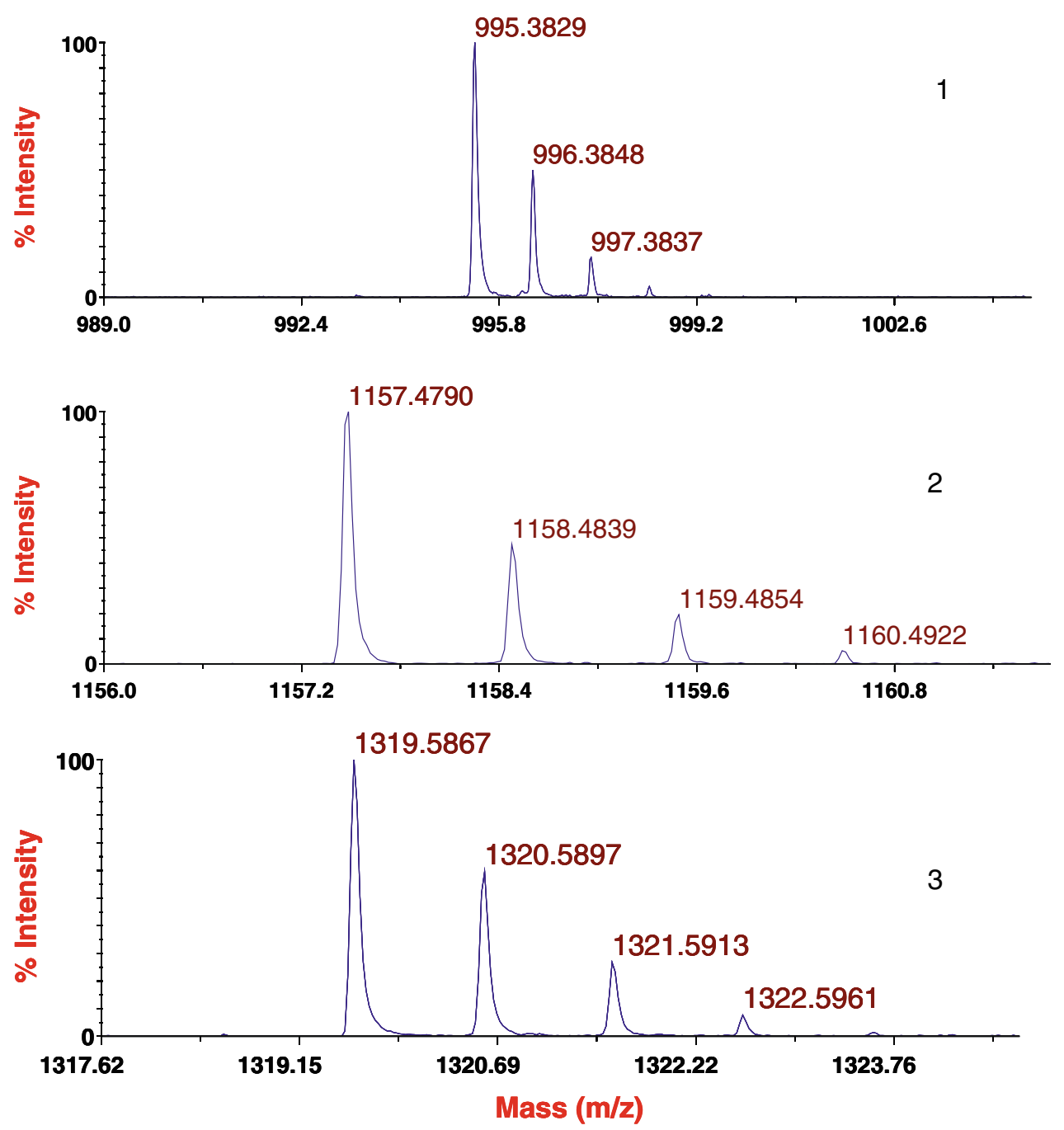

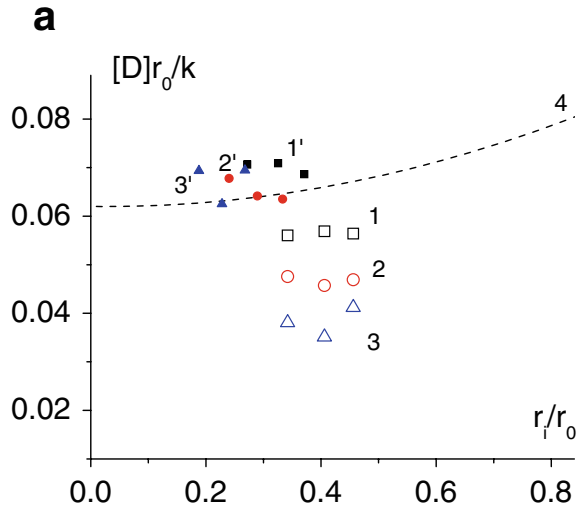

Fig. 6 Comparison of experimental hydrodynamic values (1-3) with the calculated hydrodynamic values for toroidal molecules (4): a characteristic translational diffusion coefficient $\left(1,1\right.$ : in $\mathrm{H}_{2} \mathrm{O}, 2,2^{\prime}$ : in DMF, $3,3^{\prime}$ : in DMSO) b intrinsic viscosity $\left(1,1^{\prime}\right.$ : in DMF, $2,2^{\prime}$ : in

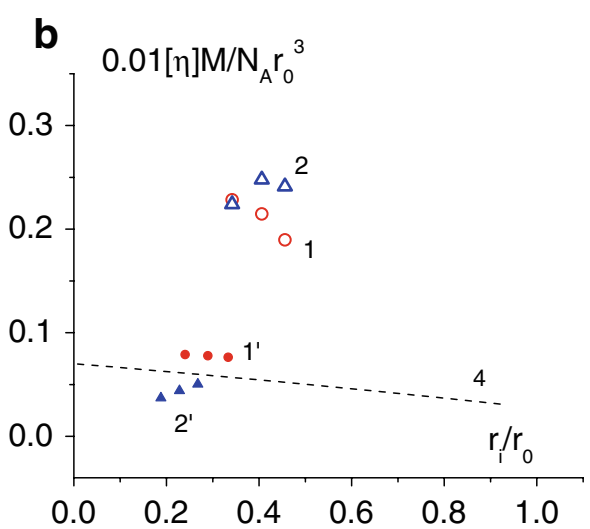

DMSO) (1-3): The hydrodynamic values are plotted in function of the space-filling (or CPK) models radii $r_{\mathrm{i}}^{\mathrm{CPK}}$ (average inner) and $r_{0}^{\mathrm{CPK}}$ (outer) of the CD molecules (Table 1). $\left(1^{\prime}-3^{\prime}\right)$ : The hydrodynamic values are plotted in function of effective outer radii $r_{0}^{\text {eff }}$ (see text) 
angles of the glycosidic links and, possibly, a twist-conformation of the sugar rings (French and Johnson 2007; Saenger et al. 1998). Nevertheless, the CD time-average characteristics, as monitored by hydrodynamic methods, can be fully interpreted by a rigid toroid model.

\section{Conclusion}

The velocity sedimentation and translational diffusion coefficients of CD molecules were measured in water, DMF, and DMSO. In DMF and DMSO also the intrinsic viscosities could be measured. It was possible to determine from the hydrodynamic data, by use of the Sedfit program, relatively accurate values for $\mathrm{CD}$ molar mass and size, despite the fact that the sedimentation coefficients were as low as 0.1-0.5 S. The correspondence of the CD hydrodynamic values with the results calculated for toroids by use of a bead-shell model is demonstrated. This comparison also shows that the outer $\mathrm{CD}$ dimensions are solvent-dependent and larger than those obtained from the crystallographic data.

Open Access This article is distributed under the terms of the Creative Commons Attribution Noncommercial License which permits any noncommercial use, distribution, and reproduction in any medium, provided the original author(s) and source are credited.

\section{References}

Allison SA (1999) Low Reynolds number transport properties of axisymmetric particles employing stick and slip boundary conditions. Macromolecules 32:5304-5312. doi:10.1021/ma990576c

Beheshti N, Bu H, Zhu K, Kjøniksen A-L, Knudsen KD, Pamies R, Hernández Cifre JG, García de la Torre J, Nyström B (2006) Characterization of interactions in aqueous solutions of hydroxyethylcellulose and its hydrophobically modified analogue in the presence of a cyclodextrin derivative. J Phys Chem B 110:6601-6608. doi:10.1021/jp056828v

Bender ML, Komiyama M (1978) Cyclodextrin chemistry. Springer, Berlin

Burckbuchler V, Kjøniksen A-L, Galant C, Lund R, Amiel C, Knudsen KD, Nystrom B (2006) Rheological and structural characterization of the interactions between cyclodextrin compounds and hydrophobically modified alginate. Biomacromolecules 7:1871-1878. doi:10.1021/bm060149o

Cantor CR, Schimmel PR (1980) Biophysical chemistry, vol 1. Freeman, San Francisco

Charlot A, Heyraud A, Guenot P, Rinaudo M, Auzely-Velty R (2006) Controlled synthesis and inclusion ability of a hyaluronic acid derivative bearing $\alpha$-cyclodextrin molecules. Biomacromolecules 7:907-913. doi:10.1021/bm0507094

Connors KA (1997) The stability of cyclodextrin complexes in solutions. Chem Rev 97:1325-1357. doi:10.1021/cr960371r

Corey RB, Pauling L (1953) Molecular models of amino acids, peptides, and proteins. Rev Sci Instrum 24:621-627. doi:10.1063/ 1.1770803

Dodziuk H (ed) (2006) Properties and application of cyclodextrins: cyclodextrins and their complexes. Wiley, USA
French AD, Johnson GP (2007) Linkage and pyranosyl ring twisting in cyclodextrins. Carbohydr Res 342:1223-1237. doi:10.1016/ j.carres.2007.02.033

Garcia de la Torre J (2001) Building hydrodynamic bead-shell models for rigid bioparticles of arbitrary shape. Biophys Chem 94:265274. doi:10.1016/S0301-4622(01)00244-7

Garcia de la Torre J, Bloomfield VA (1977) Hydrodynamic properties of macromolecular complexes. Biopolymers 16:1747-1763. doi: 10.1002/bip.1977.360160811

Garcia de la Torre J, Bloomfield VA (1981) Hydrodynamic properties of complex, rigid, biological macromolecules. theory and applications. Q Rev Biophys 14:81-139

González-Pérez A, Dias RS, Nylander T, Lindman B (2008) Cyclodextrin-surfactant complex: a new route in DNA decompaction. Biomacromolecules 9:772-775. doi:10.1021/bm7012907

Harada A (1997) Design and construction of supramolecular architectures consisting of cyclodextrins and polymers. Adv Polym Sci 133:140-191

Hedges AR (1998) Industrial applications of cyclodextrins. Chem Rev 98:2035-2044. doi:10.1021/cr970014w

Hoogenboom R, Moore BC, Schubert US (2006) Synthesis of starshaped poly(e-caprolactone) via "click" chemistry and "supramolecular click" chemistry. Chem Commun (Camb) 4010-4012. doi:10.1039/b608313g

Kratky O, Leopold H, Stabinger H (1973) The determination of the partial specific volume of proteins by the mechanical oscillator technique. Methods Enzymol 27:98-110. doi:10.1016/S00766879(73)27007-6

Lebedev AA (1930) L'interféromètre a polarisation et ses applications. Rev Opt 9:385-409

Loftsson T, Duchene D (2007) Cyclodextrins and their pharmaceutical applications. Int J Pharm 329:1-11. doi:10.1016/j.ijpharm. 2006.10.044

Longsworth LG (1953) Diffusion measurements of aqueous solutions of amino acids, peptides and sugars. J Am Chem Soc 75:5705-5709

Maechtle W, Boerger L (2006) Analytical ultracentrifugation of polymers and nanoparticles. Springer, Berlin

Nakata Y, Amitani K, Norisuye T, Kitamura S (2003) Translational diffusion coefficient of cycloamylose in aqueous sodium hydroxide. Biopolymers 69:508-509

Pavlov G, Finet S, Tatarenko K, Korneeva E, Ebel C (2003) Conformation of heparin studied with macromolecular hydrodynamic methods and X-ray scattering. Eur Biophys J 32:437449. doi:10.1007/s00249-003-0316-9

Philp D, Stoddart JF (1996) Self-assembly in natural and unnatural systems. Angew Chem Int Ed Engl 35:1154-1196. doi:10.1002/ anie. 199611541

Qi Q, Zimmermann W (2005) Cyclodextrin glucanotransferase: from gene to application. Appl Microbiol Biotechnol 66:475-485. doi: 10.1007/s00253-004-1781-5

Rinaudo M, Auzely R, Vallin C, Mullagaliev I (2005) Specific interactions in modified chitosan systems. Biomacromolecules 6:2396-2407. doi:10.1021/bm0580025

Sabadini E, Cosgrove T (2003) Inclusion complex formed between star-poly (ethylene glycol) and cyclodextrins. Langmuir 19:9680-9683. doi:10.1021/la0353273

Saenger W (1980) Cyclodextrin inclusion compounds in research and industry. Angew Chem Int Ed Engl 19:344-362. doi:10.1002/ anie. 198003441

Saenger W, Jacob J, Gessler K, Steiner T, Hoffmann D, Saube H, Koizumi K, Smith SM, Tanaka T (1998) Structure of the common cyclodextrins and their larger analogues-beyond the doughnut. Chem Rev 98:1787-1802. doi:10.1021/cr9700181

Schuck P (2000) Size-distribution analysis of macromolecules by sedimentation velocity ultracentrifugation and Lamm equation modeling. Biophys J 78:1606-1619 
Szejti J (1998) Introduction and general overview of cyclodextrin chemistry. Chem Rev 98:1743-1753. doi:10.1021/cr970022c

Szejti J (2004) Past, present, and future of cyclodextrin. Pure Appl Chem 76:1825-1845. doi:10.1351/pac200476101825

Szejti J, Osa T (eds) (1996) Comprehensive supramolecular chemistry. Vol 3 Cyclodextrins. Pergamon, Oxford
Tsvetkov VN (1989) Rigid-chain polymers. Consult Bureau, NY

Uekama K, Hirayama F, Irie T (1998) Cyclodextrin drug carrier systems. Chem Rev 98:2045-2076. doi:10.1021/cr970025p

Wenz G (1994) Cyclodextrins as building blocks for supramolecular structures and functional units. Angew Chem Int Ed Engl 33:803-822. doi:10.1002/anie.199408031 